Short report

\title{
Relapsing neurological disorder associated with rubella virus infection in two sisters
}

\author{
N. CA L L A GHA N, M. FEELY, A N D B. W A L S H \\ From the Department of Neurology, St Finbarr's Hospital, Cork, Ireland
}

SUMMARY A disseminated relapsing neurological disorder presented simultaneously in two sisters. Encephalitic features were present in one case. The illness was associated with a significaint increase in rubella specific IgM in both sisters. Despite the absence of a rubella rash, this increase would be compatible with a recent infection by the rubella virus as a basis for the illness, and the persistent elevation, with active antigenic stimulation. It is suggested that both patients might represent the clinical manifestations of perivenous demyelination caused by the rubella virus, which, in view of the relapsing nature of the illness, has progressed to plaque formation.

An increase in the serum levels of rubella specific IgM indicates active antigenic stimulation by the rubella virus, as a result of a persistent infection. This investigation is of particular value in the diagnosis of rubella infection in the absence of a rash, especially as neurological complications due to rubella are not always associated with a rubella rash (Connelly et al., 1975).

The following two cases illustrate a relapsing neurological disorder associated with a significant increase in rubella specific IgM in the absence of a rubella rash.

\section{Methods}

Rubella specific IgM in the serum was measured by the indirect fluorescent antibody test (Haire and Hadden, 1972). Rubella antibody was titrated by the haemagglutination inhibitor (HI test) by the $\mathrm{MRD}_{2}$-heparin method (Cooper et al., 1969).

\section{Case reports}

\section{CASE 1}

A 17 year old female presented with a history of flu-like illness for five days. This was followed by a headache associated with vomiting, ataxia, and diplopia. On admission to hospital, she was alert. Neurological examination showed nystagmus with a left sixth nerve palsy. Corneal reflexes were absent on both sides and the gag reflexes were dimin-

Address for reprint requests: Dr N. Callaghan, Department of Neurology, St Finbarr's Hospital, Douglas Road, Cork, Ireland.

Accepted 15 July 1977 ished. There was incoordination on finger/nose testing and with heel/shin testing. There was moderate weakness in the lower limbs associated with exaggerated tendon reflexes and bilateral extensor plantar responses. Vibration sense and position sense were absent in both lower limbs. She deteriorated over a period of two days, became drowsy and tetraplegic, with intercostal paralysis. A tracheotomy was carried out and the patient was ventilated. She was given ACTH 60 units daily initially, with a gradual decrease in the dose over a period of four weeks. During the subsequent three weeks she started to improve and was able to walk with a walking aid 10 weeks after the onset of her illness. At this stage, nystagmus was still present, but the other cranial nerve signs had subsided. There was still weakness of the arms and legs with exaggerated tendon reflexes and bilateral extensor plantar responses. Her gait was ataxic. There was no further improvement in her condition, but five months after the onset of her illness she deteriorated. She developed double vision associated with a recurrence of the left sixth nerve palsy. Her ataxia worsened and the power in both lower limbs deteriorated also. Spontaneous improvement occurred after four weeks. Eleven months after the onset of her illness she was able to get about unaided. The abnormal neurological findings at this stage were moderate weakness of both lower limbs with exaggerated tendon jerks and bilateral extensor plantar responses.

Skull and chest radiographs, pneumoventriculogram and iophendylate (Myodil) ventriculogram 
were all within normal limits. An EEG showed background activity at $8 \mathrm{~Hz}$ associated with paroxysmal activity at 6 and $4 \mathrm{~Hz}$. The CSF protein was $0.5 \mathrm{~g} / 1$ and lymphocytes $20 \mathrm{~mm}^{3}$. Rubella specific IgM was positive at a titre of $1: 10$ initially and remained positive at this level at intervals of three and 11 months after her initial illness. The rubella HI titre was 320 initially and 160 at intervals of four, 12, and 48 weeks after the initial illness. A significant CF antibody to other viral antigens was not found and virus was not isolated from the faeces.

\section{CASE 2}

A sister of case 1, a 25 year old schoolteacher, developed neurological symptoms seven days before the onset of her sister's symptoms, although she did not consult a doctor for two weeks. She first noticed clumsiness of the right hand, weakness of the right lower limb, and a sensation like a tight band over the lower part of the abdomen, together with pins and needles in the left hand. She also developed some episodes of blurring of vision. On neurological examination, she had horizontal nystagmus, weakness of grip on the right side, and exaggerated reflexes in the right arm. There was weakness of the right lower limb with exaggerated knee and ankle jerks in both lower limbs, bilateral extensor plantar responses, and absent position and vibration sense in both lower limbs. Seventy-two hours later she developed an intranuclear ophthalmoplegia, paraparesis of both lower limbs, incoordination on finger/nose testing, and transient episodes of blurring of vision. The paraparesis progressed to a total paraplegia. Over a period of two weeks she started to improve, and five weeks after the onset of her illness she was able to walk about unaided. One week later she deteriorated once more and became paraplegic with urinary retention. Improvement occurred again over six weeks, and on discharge from hospital she had minimal weakness of the lower limbs together with diminished position sense. Four months later the patient developed a right sided retro-orbital pain associated with blurring of vision and an ataxic gait. On neurological examination, she had a right sided papillitis. The visual acuity was 6/60 in the right eye and a central scotoma was found. There was also incoordination on heel/shin testing in the lower limbs with brisk reflexes and an ataxic gait. ACTH was administered in a dosage of 60 units daily initially and the dose was gradually decreased over four weeks. Her vision improved and five weeks later, the visual acuity in her right eye was $6 / 18$, with right sided optic atrophy. The scotoma had subsided as had the cerebellar signs in the lower limbs. Seven months after the onset of her illness she was entirely free from symptoms; optic atrophy was present in the right eye but neurological examination was otherwise within normal limits.

Skull radiographs, brain scan, and EEG were within normal limits. Cerebrospinal fluid protein was $0.6 \mathrm{~g} / \mathrm{l}$. The rubella specific IgM was positive in the blood at a titre of $1: 10$ initially. The titre was positive at $1: 5$ after three months, and persisted at this level at 48 weeks. The rubella $\mathrm{HI}$ titre was 80 to begin with and has remained at this level at intervals of four, 12, and 48 weeks after the initial illness. A significant CF antibody to other viral antigens was not found and virus was not isolated from the faeces.

Both parents and one sib of the patients were also investigated. Neurological examination was normal. Rubella specific IgM titre was less than $1: 5$ and rubella $\mathrm{HI}$ titre was 80 in all three.

\section{Discussion}

Encephalitis is the most common neurological complication of postnatal rubella (Miller et al., 1956). So far, 118 patients with neurological complications of rubella have been reported. In addition to encephalitis, complications include myelitis (Morris and Robbins, 1943), radiculitis, optic neuritis, and cerebral arterial thrombosis (Connelly et al., 1975). Diffuse myelitis has also been described after rubella vaccination (Holt et al., 1976). Despite the absence of a rubella rash, the increase in the blood of rubella specific IgM would be compatible with a recent infection by the rubella virus, and the persistent increase with active antigenic stimulation. The short interval between the onset of symptoms in both sisters would be compatible with recent exposure to the rubella virus in both cases. The illness was not associated with an outbreak of rubella in the area and rubella specific IgM was not raised in other members of the family. Although a reduction in the rubella $\mathrm{HI}$ titre level occurred in case 1 , it was not a feature of case 2 where the titre persisted at a level of 80 . This would not necessarily exclude a recent infection with the rubella virus. The presence of rubella specific IgM is of greater significance, as it implies active antigenic stimulation as a result of a recent or persistent infection with the rubella virus (Connolly et al., 1975). The reduction from an initial titre of $1: 10$ to a titre of $1: 5$ would suggest a recent infection.

A relapsing and remitting neurological disorder similar to multiple sclerosis due to the rubella virus has been described (Behan, 1977). Raised 
specific IgM blood levels for measles and mumps have been documented in patients with multiple sclerosis (Millar et al., 1971), suggesting an association between multiple sclerosis and certain viruses. Perivenous demyelination is a feature of the acute cerebral complications of rubella, measles, and varicella. Mixed cases showing the pathological changes of both multiple sclerosis and perivenous encephalomyelitis have been mentioned (McAlpine et al., 1972), and it has been suggested that the mechanisms which initiate multiple sclerosis and encephalomyelitis are probably identical (McAlpine et al., 1972). Behan (1977) states that the difference between encephalomyelitis and multiple sclerosis results from genetic and immunological factors which regulate the patient's response to a common agent. The illness in case 2 would be compatible with a clinical diagnosis of multiple sclerosis, and in patient 1 there was evidence of a disseminated neurological disorder in which one relapse occurred. Encephalitic features were also present during the initial stages of her illness. In the absence of a specific diagnostic test for multiple sclerosis, it is impossible to distinguish between multiple sclerosis and a relapsing and remitting self-limiting illness caused by the rubella virus. However, the possibility that the patients represent the clinical manifestations of a process of perivenous demyelination which has progressed to plaque formation cannot be excluded.

Both cases illustrate the value of the application of immunoglobulin titres to specific viruses in the investigation of neurological disorders, and confirm previous observations that neurological complications associated with recent infection by the rubella virus may occur in the absence of a rash.

We wish to thank Dr J. Connelly of the Department of Microbiology and Immunology, Queen's University Belfast for the viral studies.

\section{References}

Behan, P. (1977). Diffuse myelitis associated with rubella vaccination. British Medical Journal, 1, 166.

Connelly, J. H., Hutchinson, W. N., Allen, I. V., Lyttle, J. A., Swallow, M. W., Dermott, E., and Thompson, D. (1975). Carotid artery thrombosis, encephalitis, myelitis and optic neuritis associated with rubella virus infection. Brain, 98, 583-594.

Cooper, L. Z., Matters, B., Rosenblum, J. K., and Krugman, S. (1969). Experience with a modified rubella hemagglutination inhibitor antibody test. Journal of the American Medical Association, 207, 89-93.

Haire, M., and Hadden, D. S. M. (1972). Rapid diagnosis of rubella by demonstrating rubella specific IGM antibodies in the serum by indirect immunofluoresence. Journal of Medical Microbiology, 5, 237-242.

Holt, S., Hudgins, D., Krishnan, K. R., and Critchley, E. M. R. (1976). Diffuse myelitis with rubella vaccination. British Medical Journal, 2, 1037-1038.

Millar, J. H. D., Fraser, K. B., Haire, M., Connelly, J. H., Shirodaira, P. V., and Hadden, D. S. M. (1971). Immunoglobulin $M$ specific for measles and mumps in multiple sclerosis. British Medical Journal, 2, 378-380.

Miller, H. G., Stanton, J. B., and Gibbons, J. L. (1956). Parainfectious encephalomyelitis and related syndromes; a critical review of the neurological complications of certain specific fevers. Quarterly Journal of Medicine, 25, 427-505.

Morris, M. H., and Robbins, A. (1943). Acute infectious myelitis following rubella. Journal of Pediatrics, 23, 365-367.

McAlpine, B., Lumsden, C. E., and Acheson, E. D. (1972). Multiple Sclerosis, a Reappraisal. Second edition. p. 517-519. Churchill Livingstone: Edinburgh and London. 